\title{
La dramaturgia musical en el litoral. Una propuesta de estudio internacional
}

\author{
Aníbal Cetrangolo ${ }^{\bullet}$ \\ IMLA. RIIA. Universidad de San Martin •
}

\begin{abstract}
Resumen
Se propone en este texto un examen comparativo referido a la actividad de teatro musical en el litoral argentino. El objeto lírico convoca al estudio interdisciplinario en cuanto el mismo reúne la actividad no solo de músicos sino también de literatos, artistas de lo visual y artesanos de profesiones muy específicas. Su estudio, en la Argentina de fines de siglo XIX y principios del siglo XX convoca necesariamente el examen de las migraciones. El registro de la historia de la ópera en el interior, desgraciadamente, es tema que, salvo muy pocas excepciones se circunscribe a la crónica circunstancial, motivada por lo general a la inauguración de algún teatro. Los archivos teatrales son exiguos: los programas de sala no fueron conservados de manera sistemática y no fueron elaborados elencos de espectáculos. Estos teatros más que producir espectáculos, fueron receptáculo de troupes itinerantes. Los pocos documentos conservados dan cuenta, en efecto, de la presencia de las compañías y de los miembros que las integraban. Es imprescindible el estudio de las publicaciones periódicas para reconstruir un pasado muy vital. Es solo la comparación de esos datos provenientes de las diferentes salas del área lo que puede dar una idea de los itinerarios de esos grupos. Para hacer posible un estudio científico del tema y conocer el real espesor de esas producciones se impone, a su vez, el cotejo de los documentos provenientes de los teatros de la zona con los datos provenientes de las salas externas a ella, tanto de Buenos
\end{abstract}

\footnotetext{
- Nació en Buenos Aires. Es Doctor en Musicología (Ph. D.) por la Universidad de Valladolid. Dirige el Istituto per lo studio della Musica Latinoamericana (IMLA) que fundó con F.C. Lange. Sus investigaciones sobre Giacomo Facco fueron publicadas en cuatro volúmenes y sus estudios sobre las migraciones de la ópera en otros dos. Coordina el study group Relaciones Italo Iberoamericanas de la I.M.S. Diploma al Mérito Konex en musicología. Es docente de la Universidad Nacional de San Martin.

- Investigación en curso realizada por el grupo RIIA (Relaciones Italo-Iberoamericanas, International Musicological Society, Universidades Cà' Foscari Venezia-Cambridge-Valladolid-República del UruguayUniversidad de San Martin), en colaboración con el proyecto trienal sobre Historia y Patrimonio de la Argentina Moderna, dirigido por Fernando Devoto (PICT n²015-3831, «Historia y patrimonio de la Argentina moderna. Inmigración, transferencia y readaptación de saberes en las dimensiones simbólicas y materiales de los teatros del litoral rioplatense y su conservación», FONCYT 2016-2019). El proyecto comprende el examen de cinco teatros líricos construidos en las riberas del río Paraná en las ciudades de San Nicolás de los Arroyos, Zarate, Paraná, Gualeguay y Goya.
} 
Aires como del exterior del país. Se describe la conformación de una base de datos ya activa hace decenas de ańos sobre estos temas y la incorporación a la misma de los datos provenientes de los teatros del litoral argentino.

Palabras clave

Ópera · Migraciones · Litoral · Argentina

\begin{abstract}
In this text, we propose a comparative study of the musical theatrical activity of the «littoral region» of Argentina. Given the lyrical object of our study, we will attempt an interdisciplinary analysis that will include the craft of musicians, writers, visual artists and artisans with very specific professions. Likewise, we will study the migrations in Argentina around the end of the nineteenth century and the beginning of the twentieth century. The recording of the history of the opera in upstate areas of the country is, unfortunately, circumscribed to (with a few exceptions) the forms of circumstantial chronicles motivated by the opening of some new theatre. These theatre archives are however exiguous since theatre programs and information regarding the casts were not preserved systematically and the theatres did not really produce shows but rather hosted visiting theatre companies. The very few surviving documents attest to the existence and the members of these companies, hence, the importance of studying periodic publications. The study of these materials is vital to reconstruct the past and we gather this can be achieved by comparing data coming from different playhouses, which could in turn help guesstimate the itineraries of the aforementioned theatre companies. Similarly, to carry out scientific research on this issue and to learn about the real incidence of these theatrical productions, it is also necessary to draw a comparison between documents descriptive of nearby theatres with data pertaining to playhouses located in outer areas, that is, Buenos Aires and other parts of Argentina. This said, we hereby describe the configuration of a database that has been in active use for decades on these very complexities and the incorporation of new data regarding theatres from the «littoral region» of Argentina.
\end{abstract}

\title{
Key words
}

- Opera · Migrations · the «littoral region» Argentina 
Este texto ambiciona estimular el estudio de la penetración lírica en el interior del continente. Interesa aquí analizar el periodo de las grandes migraciones que a él aprodaron entre fines del siglo XIX y principios del siglo XX. Este fenómeno ha influido en la determinación de los perfiles de esas comunidades que han acogido tanto a personas como objetos culturales y esto ha sucedido por dos motivos: por un lado, porque gran parte de los actuales habitantes de la región reconocen en aquellos viajeros a sus propios ancestros y por otro, a causa de la poderosa potencialidad de la ópera de penetrar en la vida cotidiana de las personas, incluso de aquellas que jamás la han conocido en el teatro ${ }^{1}$. El interés sobre estos aspectos, por eso, no puede limitarse al mero ámbito académico en cuanto compromete al perfil identitario de la población. En este sentido, la comunidad entera es destinataria de estas páginas; por ese motivo algunas de las consideraciones que siguen resultarán tal vez triviales al estudioso especializado.

Durante el periodo en estudio, en el interior del territorio sudamericano, fueron construidos un número muy considerable de teatros. Gran parte de ellos, sobre todo en el caso de Argentina y Uruguay, fueron promovidos por sociedades de inmigrantes. La arquitectura de esas salas, su denominación, las manifestaciones proyectuales, indican un primer destino de esos teatros: la producción operística, lo que es notable en comunidades que poco tiempo antes de la edificación de esas salas habían debido afrontar problemáticas primarias. Puedo entonces cerrar este párrafo con la frase inicial del mismo, pero corrigiéndola con convicción: «durante el periodo en estudio, en el interior del territorio sudamericano, fueron construidos un número muy considerable de teatros líricos».

Dejando provisoriamente de lado la explicación de aquel particular vínculo de la ópera con aquellos migrantes predominantemente analfabetos ${ }^{2}$, he de subrayar algunas características de un género que lo hace tan apto al desplazamiento. La ópera, en efecto, se define como objeto eminentemente trashumante y por ende transnacional ${ }^{3}$. Esa internacionalización hace que ella domine un territorio que no tiene una capital estable. Su centro es variable y cualquier periferia de hoy puede resultar el centro de mañana. Los habitués de la ópera bien saben que el «centro del mundo» en 1951 fue México cuando Callas cantó allí Aida o en 1969 resultó Buenos Aires cuando Price y Bergonzi se encontraron en un Trovatore mítico. Por cierto, hay sedes históricas —ineludiblemente La Scala, con sus innumerables estrenos célebres - pero para la ópera, siempre vigente, cuenta la luz del presente.

\section{La investigación}

Ese aspecto móvil de lo lírico hizo considerar, hace no demasiado tiempo a John Roselli, que para estudiar con provecho el fenómeno operístico era imprescindible examinar los archivos del Teatro Colón. Es relevante subrayar, a propósito de su excepcional personalidad, que Rosselli, aunque devoto de la ópera lírica, no era musicólogo de profesión, sino más bien 
un estudioso de la historia de la cultura. Él se colocaba en las antípodas de las arrogantes firmezas de las ciencias establecidas y por eso fue un convencido representante de los estudios interdisciplinarios; desde 1964 encontró una colocación académica ideal en la Universidad de Sussex, un ateneo fundado poco antes y que, precisamente promovía enfoques de apertura científica. Fue recién desde la década del 1980 — Rosselli tenía más de cincuenta años- que el estudioso publicó los textos que habrían de resultar fundamentales para el nuevo curso de los estudios del teatro musical y que recorrían un terreno casi intransitado hasta entonces por la academia: la historia económica de la ópera ${ }^{4}$. Precisamente son esos años los que pueden considerarse fundacionales de una nueva musicología italiana. Estas dinámicas peninsulares no fueron en absoluto ajenas a los estímulos de Rosselli. En efecto, es del 1984 un texto de Lorenzo Bianconi y Thomas Walker que por muchos motivos puede considerarse signo de un cambio de ruta respecto de los estudios anteriores 5 . Siempre en esos ańos, estos nuevos enfoques referidos al teatro lírico fueron nutridos por un grupo de investigadores —italianos y no- que idealmente fueron convocados en una obra imprescindible sobre estos temas: $\mathrm{La}$ Storia della Opera Italiana, coordinada por Bianconi y Giorgio Pestelli. En cuanto la mayoría de estos autores centrales para estos estudios, son desconocidos por la academia argentina, a riesgo de fatigar al lector, creo necesario al menos nombrarlos: el grupo comprende además de Rosselli, al célebre Carl Dahlhaus y a los ya citados Bianconi y Pestelli, pero también a estudiosos como Franco Piperno, Fiamma Nicolodi, Fabrizio Della Seta, Elvidio Surian, Sergio Durante, Mercedes Viale Ferrero, Gerardo Guccini, Kathleen Kuzmich Hansell, Renato Di Benedetto, Paolo Fabbri, Marzio Pieri, Roberto Leydi y Giovanni Morelli. Ellos se han ocupado de la ópera con nueva metodología y cubriendo un abanico de intereses de una riqueza desconocida antes; en una pluralidad que es, por cierto, impuesta por el carácter típicamente multifacético del teatro lírico. Fue comprendido inmediatamente cómo era el propio objeto de estudio el que convocaba a estudiosos de literatura, de la economía, de la plástica y de la arquitectura teatral con idéntica legitimidad y es por eso que fueron convocados trabajos interesados en aspectos como el estudio del sistema productivo — tan afín a los estudios de Rosselli-, el papel de los diferentes actores del espectáculo —el libretista, el compositor, el cantante- el examen del lugar teatral, el estudio de la dirección escénica, la presencia del baile en el melodrama, la dramaturgia y la teoría de lo lírico, los institutos métricos y formales del texto, el contacto con la literatura, la difusión operística en los estratos populares de la sociedad y la 
función de la ópera en cuanto emblema de la nación italiana. Una generación sucesiva de valiosos estudiosos italianos continúa estos intereses con gran provecho; entre estos nombro al menos a Michele Girardi, Emanuele Senici, Emilio Sala y Marco Beghelli.

\section{El grupo RIIA de la IMS}

Estos nuevos estudios, que han dado especial énfasis al análisis de los aspectos de producción y circulación del espectáculo, han estimulado el examen de las migraciones de la ópera. Un particular grupo de investigadores se interesó especialmente en los viajes atlánticos del género y se propuso recabar datos sobre las tournées que, procediendo de Europa, seguían la ruta hacia las Américas. Se trataba de la misma vía que, contemporáneamente, hablamos de fines del siglo XIX, recorrían tantos migrantes. Fue así que, en 1992, en ocasión de uno de los encuentros quinquenales de la International Musicological Society, se formó un específico grupo de estudio de la I.M.S., el equipo RIIA (Relaciones Ítalo Iberoamericanas. El teatro Musical) ${ }^{6}$. Desde entonces RIIA estudia las actividades de los teatros líricos edificados sobre las ciudades puertos de aquel trayecto como Río de Janeiro, Montevideo, Buenos Aires, concentrándose sobre todo en la circulación de títulos y artistas que se transfirieron por esa vía marítima. Estos estudios, ya desde el comienzo de las labores, impusieron al grupo la necesidad de ordenar las informaciones no solamente en los lugares de llegada sino también en los de salida. Como abajo se explicará, para poder actuar un estudio relacional, resultó imprescindible forjar una herramienta que pudiese acoger con un formato homogéneo la masa de datos provenientes de fuentes muy dispares. Casi inmediatamente fue claro que del cotejo de datos surgían cuestiones del mayor interés científico. Así resultó que esos esfuerzos por estudiar la opera en tránsito han generado, diferentes productos musicológicos que fueron presentados en numerosas publicaciones y reuniones científicas del grupo. En tiempos más recientes, y de manera muy específica desde $2009^{7}$, el equipo ha afrontado un nuevo desafío: examinar la penetración lírica en el continente. La vocación «acuática» de la ópera ${ }^{8}$ nos hizo considerar in primis el aprovechamiento de las vías navegables. Esta estrategia, ya recorrida con éxito por Xoan Manuel Carreira en su examen de la penetración de la ópera italiana en Iberia durante el siglo $\mathrm{XVIII}^{9}$, es en este caso estimulada por la presencia de la gran cantidad de teatros edificados en las riberas de los ríos Paraná y Uruguay. La difusión fluvial no fue por cierto la única y el grupo en absoluto se exime del examen de tantas importantes salas que fueron construidas en centros urbanos surgidos junto a la red ferroviaria o que se desarrollaron en torno a algún tipo de explotación de gran interés económico. 


\section{Obstáculos}

Siempre recordando el afán proselitista de este escrito y con el propósito de transferir nuestra experiencia a los estudiosos que convocamos a nuestras filas, creo útil reflexionar sobre los mayores obstáculos que hemos debido superar en estos años. Ellos no son insuperables, pero deben ser conocidos para que puedan ser afrontados. Como se verá, algunos parecen de una nimiedad desconcertante y son los más insidiosos porque resultan difíciles de detectar en su peligrosidad.

Una primera dificultad a sortear es la de cierto inmovilismo, cierta resignación, marcados por algunas convicciones acendradas y difundidas en la sociedad en general. Ideas del tipo «todos los italianos son musicales» $\mathrm{o}$ «aquí no se ha conservado nada» son realmente perniciosas en cuanto generadoras de prejuicios. Solamente un examen científico puede echar luz a zonas dominadas por el sentido común.

Pero cuando se abandona la calle y se penetra en la academia las cosas tampoco resultan simples; los desafíos son otros. La musicología es una ciencia relativamente reciente y las fuerzas de estos estudios en nuestros países son, si bien a menudo valiosas, también muy escasas. La producción científica en el terreno humanístico, por otro lado, y como no podía ser de otra manera, ha sido gobernada por pasiones políticas que si bien son de diferente signo, en general resultan identificadas por un común denominador de tipo nacionalista. Como consecuencia, tanto los estudiosos de lo étnico como los analistas de la música académica se han interesado de forma casi exclusiva por la música producida por gentes nacidas en el país. Si una actitud análoga hubiese sido adoptada, por ejemplo, por la musicología estadounidense, los colegas norteamericanos se habrían concentrado en el estudio de los cheyennes, el jazz o la obra de Samuel Barber y habríamos perdido los ensayos fundamentales de Philip Gossett sobre Rossini o de Richard Taruskin sobre Chaikovski. Esta actitud científica que aquí señalo es llevada a menudo a los extremos porque ni siquiera son estudiados los efectos locales de la música que ha llegado a estas tierras. Prueba de esto es que en nuestras universidades es mucho más sencillo encontrar tesis sobre la cumbia villera que sobre la recepción local de la música de Brahms, un músico que no es visto como del patrimonio de todos sino «de los alemanes». Es notable que se actúe sobre los productos musicales europeos un rechazo que no afecta a otros objetos culturales llegados de fuera como la comida: así resulta que los impulsos de narcisismo local han permitido a los argentinos apropiarse de la pizza — pronunciado infaliblemente pixa-, "que-es-mejor-que-la-italiana». Esto no ha ocurrido con un fenómeno musical tan difundido como la ópera, por ejemplo, que de forma curiosa es considerada, en Argentina, objeto elitista, extranjero, superado.

Por supuesto, escapan a esta lógica los muy valiosos esfuerzos que algunos estudiosos realizan en el examen de los enclaves de migrantes en Argentina ${ }^{10}$, y este grupo comprende algunos valiosos musicólogos ${ }^{11}$; de todas maneras, resulta aún arduo mostrar cuánto es la sociedad general, toda la sociedad, la que ha sido conformada en la asimilación — a menudo muy peculiar— de fenómenos llegados de fuera. Como consecuencia de estas elecciones generales y académicas resulta que el estado de conservación de los materiales documentales que se refieren a estos estudios es crítico. El registro de la historia de la ópera en las localidades del interior es, desgraciadamente, un tema que, salvo muy pocas excepciones, se circunscribe a la reiteración rutinaria de los escasísimos datos. Estas pocas infor- 
maciones son recordadas por el periodismo en las conmemoraciones, normalmente los aniversarios de la inauguración del teatro local. Sin embargo, hay muchísimo por hacer. Los teatros, si bien más que producir espectáculos fueron receptáculo de troupes itinerantes, representaron el fulcro de una actividad muy dinámica. La realidad de un teatro es lo que él ha presentado entre sus paredes, es decir la producción de espectáculos que esos sitios han sido capaces de organizar en su devenir. La documentación prínceps para este tipo de estudio consiste en los afiches y programas de sala; allí consta la fecha del espectáculo, el título y el elenco, además de otras informaciones en absoluto secundarias como las publicidades que dan un fiel reflejo de toda una sociedad. Muy pocos teatros argentinos conservan esos materiales. En alguno de los casos, incluso salas de importancia, orgullosas de su belleza externa pero indolente respecto de la propia historia, conservan la famosa «caja de papeles viejos» donde entre documentos varios es posible dar con algún programa. Remedando una antigua comedia de Lope, esos mínimos materiales son conservados en un huerto que es inaccesible a quien es ajeno al Círculo dirigente. Los años pasan, los materiales, obviamente corren peligro; no se investiga ni se deja investigar. La situación es más alarmante cuando se percibe que no existe una presión adecuada de parte de la sociedad o de los especialistas para que este estado de cosas cambie y provocar que los custodios muestren al público un material que es de interés general. Esta actitud, sobre todo la de las gentes más conscientes, las de la academia, es importante. Ya no podemos hablar de descuido u olvido. Es imprescindible profundizar el mecanismo que ha conducido a ciertas elecciones para que este pueda ser modificado.

En la práctica operativa es obligado señalar otras dificultades que han retardado e incluso impedido en estos ańos no pocas de las iniciativas del grupo. Me siento obligado a describir esta situación ya que encuentro imprescindible extraerla de su oscura banalidad. Ocurre que en un mundo que se pretende globalizado e hiper comunicado no pocas veces un factor que ha atentado a nuestros proyectos con potencia destructiva ha sido la deficiencia de las comunicaciones, lo que, en un grupo como el nuestro, constituido como red difundida en el espacio, resulta letal. Es inútil contar sobre nuestro escritorio con la facilidad del mail, de Skype, de WhatsApp o de Facebook si esas herramientas no se usan y los mensajes no reciben respuesta. Por experiencia sé que son las personas más ocupadas —el vicerrector de la Universidad de Venecia, por ejemplo- quienes responden de manera inmediata a cualquier solicitud y que por otro lado son a menudo los jóvenes estudiantes de nuestro grupo que ponen a prueba la paciencia más tenaz. Aconsejo a estas personas y al lector en general consultar algún epistolario del siglo XVIII o XIX; se enterarían así que, por ejemplo, entre el 6 y el 11 de julio de 1889, Verdi - a la sazón en Montecatini- y su libretista Boito —que estaba seguramente en Milan - se intercambiaron al menos cuatro cartas.

Me atrevo a lanzar estas denuncias en esta sede, con la esperanza que también esto sea objeto de análisis. Estoy convencido que cualquier hecho social puede ser relevante para el estudio científico, incluso los mails que tardan en contestarse o el uso instrumental de la historia por parte de profesionales del área. Un examen de estos fenómenos que evite soluciones voluntaristas, podría modificar una situación hecha rutina y, sobre todo ayudar a comprender elecciones profundas, no conscientes, que han provocado — según creo — un progresivo degrado, la efectiva perdida de la identidad comunitaria. 


\section{Cronologías}

En general los teatros, al menos los que respetan la propia historia, no solo atesoran sus programas con orgullo, sino que organizan esos documentos y elaboran listas de los espectáculos posiblemente desde el momento inaugural. Para el RIIA estos materiales son preciosos: es precisamente la comparación de los elencos de títulos y artistas presentados en los diferentes teatros lo que permite establecer la circulación de óperas y operadores e intentar una evaluación de esas actividades. Se considere que otra premisa en el estudio es la de evitar la mera actitud descriptiva de los fenómenos locales. La realidad de un teatro es comprendida en función de la comparación con otras situaciones y en ese sentido no hay que temer el cotejo con lo que aparece lejano: «lo que necesitamos es buscar relaciones sistemáticas entre diversos fenómenos, no identidades sustantivas entre fenómenos similares» (Geertz, 2003: 51); es decir, para medir la real relevancia de los espectáculos en Zarate es esencial entender que es lo que sucedía en centros cercanos como San Nicolás de los Arroyos, pero tambien con la realidad europea.

Cuando nuestro grupo se abocó a estos cotejos, la tarea mostró inmediatamente sus dificultades: resultaba ímprobo realizar el estudio comparativo por algunos motivos fundamentales: por un lado, no es simple dar con un reservorio capaz de reunir en una misma sede las cronologías, en gran parte no digitalizadas, del Teatro della Scala, del Teatro San Carlo de Nápoles, del Teatro Municipal de Rio de Janeiro, del Teatro Solís, etc. Por otro lado, resulta que cada uno de esos materiales fue elaborado con criterios editoriales propios y diferentes. El grupo RIIA comprendió que era fundamental encontrar alguna vía para homogeneizar los datos y albergarlos en un continente digital único. Para ello ha elaborado una base de datos con informaciones recabadas de los teatros de la ruta atlántica. Esa base consta de diferentes secciones — tablas - que se refieren a las características de los lugares teatrales, de los datos de los operadores musicales y de los títulos que fueron presentados. Estos dos últimos aspectos son los más socorridos por los estudios y en la actualidad llegan a más de 30.000 registros. Obviamente, esta labor iniciada hace veinte años se encuentra en constante actualización y es ambición del grupo poner estos materiales a disposición del público en general a través de una plataforma informática. Esta herramienta ha permitido dar cuenta de itinerarios de títulos y recorridos de cantantes que eran absolutamente desconocidos antes y tales datos han resultado estímulo de numerosas tesis de doctorado que fueron dirigidas por miembros del RIIA.

El caso argentino representa una dificultad particular, derivada del señalado descuido en la conservación del documento programa: muy pocos teatros han elaborado ese tipo de cronologías que dan cuenta de la vida cultural de una comunidad. Así el caso de Buenos Aires, que contaba con al menos siete salas que regularmente ofrecían ópera; allí fueron elaboradas solamente tres cronologías: las del Teatro Colón y las de dos salas — una de ellas muy secundaria-: el teatro Coliseo y el teatro Doria, que luego se llamó Marconi. No existen listas de este tipo capaces de registrar la historia de sedes centrales de la ciudad como como el primer teatro Colón —inaugurado en abril de 1857-y tanto menos del Teatro 
de la Opera, que fue la sala lírica central de la ciudad desde 1888 — cuando aquel Colón fue abatido- hasta la inauguración de la nueva sala en 1908. Aún más, la empresa del investigador se vuelve aun casi desesperante al constatar que la mayoría de los teatros ni siquiera conserva los programas de salas o los afiches de sus propios espectáculos. Por cierto, no se trata de elaborar la apología acrítica de este tipo de documentos; ellos deben ser estudiados con prudencia porque el anuncio impreso no es más que una promesa. Los amateurs bien saben cuántos resfríos o caprichos de los divos han provocado el reemplazo a último momento de un cantante o incluso la supresión de un espectáculo. Un programa o una fecha necesita de un cotejo con otras fuentes, como la información periodística, lo que no quita que tales documentos resultan de importancia muy relevante.

\section{Oportunidades y la falta de programas}

Como los programas se imprimían en tiraje considerable, muchos de ellos son atesorados entre las viejas fotos por personas en archivos privados como testimonio de alguna velada de ópera en la que participaron los abuelos. Los programas, además, son también objeto de pulsión fetichista y son acumulados por coleccionistas que resultan a menudo celosos de su propiedad. Esta última actividad ha favorecido un mercado caótico, sobre todo en internet, de ejemplares aislados de estos materiales. Por cierto, estos ejemplares aislados son eslabones y solamente injertados en la cadena que supone la colección pueden permitir el estudio sistemático.

\section{Acciones de la sociedad}

Si bien la situación descripta, puede llevar a una justificada desazón, la ignavia que se ha señalado en las instituciones puede provocar reacciones positivas en la sociedad. Como sucede con tantas áreas críticas, cuyo descuido por parte del establishment han provocado acciones por parte de la sociedad — como los temas ecológicos, por ejemplo- una nueva conciencia de los ciudadanos podría revertir este escenario nefasto. Es importante que cada persona considere el valor del pasado familiar reconociendo la importancia del propio patrimonio de documentos; esa pequeña memoria merece ser conservada y compartida en una operación de profundo sentido ético. Los desvanes familiares son un archivo diseminado en las casas de Zárate o Santa Fe. Es muy probable que allíse encuentren documentos únicos, como así también que algunas personas mayores conserven memorias orales insustituibles. En nuestro tema musical habrá que aprender mucho de operaciones de este tipo que se han desarrollado en sitios como Génova en la recuperación de los diarios de vida de los migrantes o en Santa Fe con el proyecto 
del Portal Virtual de la Memoria Gringa de la Universidad del Litoral $^{12}$. Los científicos nos pueden dar pautas de método insustituibles, pero es preciso recordar que es desde las cátedras que se ha decidido estudiar algunos temas y relegar otros. En la deseada ecología de la memoria que debe ser compartida por toda la sociedad se puede partir de una premisa: la historia es demasiado importante para abandonarla a los historiadores.

\section{Acciones de la comunidad científica}

Más allá de esta operación difusa, el rol del especialista es insustituible. Con relación a la reconstrucción de las actividades es posible — ¿hasta cuándo? - recurrir a las publicaciones periódicas que anuncian la próxima llegada de una troupe, la critican después del espectáculo o la promueven a través de anuncios pagados. El estudio de esos datos escondidos en la que tal vez sea la última copia supérstite de un periódico en archivos descuidados es decisivo para reconstruir un pasado muy vital.

Aquí la labor de los investigadores, sobre todo de los estudiosos locales, es insustituible porque solamente ellos pueden monitorear in situ la dispersa red de archivos públicos y privados, la existencia de publicaciones con escasa difusión que en algunos casos han tenido una vida breve. La exterminada red de asociaciones de inmigrantes que cubre un país como Argentina representa también una vastísima trama de pequeñas bibliotecas y archivos que conservan revistas y diarios vinculados a esa específica comunidad; tal vez en un único ejemplar y en estadio absolutamente precario. La inmediata digitalización de esos materiales es tarea que debería interesar incluso a importantes instituciones que los países de origen de esos migrantes mantienen en Argentina, me refiero a los Istituti Italiani di Cultura, en primer lugar, o a las empresas extranjeras que residen en el país. La actual tecnología permite, con gastos muy contenidos, elaborar estas tareas imprescindibles.

\section{Conclusión}

Para hacer efectiva la participación a estas actividades tanto de especialistas como de la ciudadanía general, RIIA está organizando núcleos abocados a estas tareas en diferentes ciudades argentinas, uruguayas y brasileras. Uno de estos grupos es patrocinado por la Universidad del Litoral y es coordinado por Adriana Crolla. El referente de este grupo es Gaspar Bertoni, cuya referencia es la siguiente: gasparbertoni@hotmail.com. Asimismo las personas o instituciones interesadas en participar en este proyecto pueden dirigirse también a la siguiente dirección de correo electrónico: aecetrangolo@gmail.com y consultar la página informática www.imla.it. 


\section{Notas}

${ }^{1}$ Cetrangolo, A.E. (2018b)

${ }^{2} \mathrm{He}$ tratado de explicar cómo incluso personas provenientes de sitios carentes de salas líricas y deficitarias de educación formal conocían música y texto de las óperas: Cetrangolo (2018b).

${ }^{3}$ Cetrangolo. A.E. (2015).

${ }^{4}$ Fundamentalmente The Opera Industry In Italy From Cimarosa To Verdi: The Role Of The Impresario, (1984) y Singers Of Italian Opera: The History of a Profession (1992).

${ }^{5}$ Bianconi, L. y Walker, T. (1984).

${ }^{6}$ El grupo es coordinado por el Istituto per lo Studio della Musica Latinoamericana, fue formado en 1992 y su chair es Aníbal Cetrangolo. ${ }^{7}$ Si bien RIIA se interesó por la migración lirica en el interior del continente desde diez años antes, fue desde 2009 que el grupo colaboró de manera institucional aportando informaciones históricas con el equipo organizado por TAREA, Instituto de Investigaciones sobre el Patrimonio Cultural de la UNSAM que tuvo como objeto la restauración del telón de boca del Teatro del Círculo de Rosario, Argentina.

${ }^{8}$ Cetrangolo, A.E. (2018a) y (2004).

${ }^{9}$ Carreira, Xoan M. (1990), (1996) y (2003).

${ }^{10}$ Es el caso de estudiosos como Fernando Devoto, Alicia Bernasconi, Adriana Crolla y otros.

${ }^{11}$ Así los estudios de Fátima Graciela Musri (2004), como Músicos inmigrantes. La familia Colecchia en la actividad de San Juan 1880-1910 y los recientes trabajos sobre el tema de Silvina Luz Mansilla e Ignacio Weber.

${ }^{12}$ http://www.fhuc.unl.edu.ar/portalgringo/crear/gringa/portal_i.html

\section{Referencias bibliográficas}

Bianconi, L. y Walker, T. (1984). «Production, Consumption and Political Function of Seventeenth-Century Opera». Early Music History, 4, 209-296.

Carreira, X M. (2003). «L'impresario Setaro» en Il teatro dei due mondi. Padua: Imla.

(1990). «El teatro de ópera en la Península Ibérica (hacia 1750 1775). Nicola Setaro» en De musica hispana et aliis. Miscelánea en honor del Profesor Doctor José López Calo. Santiago de Compostela: Universidad de Santiago de Compostela, pp. 27-117.

_ (1996). «Il balletto nella penisola iberica e nei paesi latinoamericani» en Basso, A. (coord.) Musica in scena. Storia dello spettacolo musicale. Vol. V. Turín: Utet, pp. 661-697.

Cetrangolo, A.E. (2018a). "Ríos vs. fronteras», Amérique Latine Histoire et Mémoire (35). [En línea] consultado 14-11-218 en http:// journals.openedition.org/alhim/

(2018b). Dentro e fuori il teatro. Ventura degli italiani e del loro melodramma nel Rio de la Plata. Isernia: Iannone editore. 
(2015) Opera, barcos y banderas, El melodrama en la inmigración italiana de la argentina (1880-1920). Madrid: Biblioteca Nueva.

(2003). «Opera y Puerto». Opera e Vigo, (4-5), 9-22. Vigo: Instituto de Estudios Vigueses.

Geertz, C. (2003). La interpretación de las culturas. Barcelona: Gedisa. Musri, F.G. (2004). Músicos inmigrantes. La familia Colecchia en la actividad de San Juan 1880-1910. San Juan: EFFHA.

Rosselli, J. (1992). Singers OfItalian Opera: The History of a Profession. Cambridge: Cambridge University Press.

(1984). Opera Industry In Italy From Cimarosa To Verdi: The Role Of The Impresario. Cambridge: Cambridge University Press.

\section{Cetrangolo, Aníbal}

«La dramaturgia musical en el litoral. Una propuesta de

estudio internacional». El hilo de la fábula. Revista anual del Centro de Estudios Comparados (19), 189-200. 